Revista Brasileira de Cartografia

ISSN 1808-0936 | https://doi.org/10.14393/revbrascartogr

Sociedade Brasileira de Cartografia, Geodésia, Fotogrametria e Sensoriamento Remoto

\title{
Análise Comparativa das Normas de Controle de Qualidade Posicional de Produtos Cartográficos do Brasil, do INCRA e da ASPRS
}

\section{Comparative Analysis of Positional Quality Control Standards for Cartographics Products in Brazil, INCRA and ASPRS}

Lígia da Silva Barbosa1 ${ }^{1}$, Larissa Messias de Souza, ${ }^{2}$, Marcelo José Pereira da Cunha ${ }^{3}$ e Afonso de Paula dos Santos 4

1 Universidade Federal de Viçosa, Departamento de Engenharia Civil, Viçosa, Brasil. ligia.barbosa@ufv.br ORCID: https://orcid.org/0000-0003-1929-8110

2 Universidade Federal de Viçosa, Departamento de Engenharia Civil, Viçosa, Brasil. larissa.messias@ufv.br ORCID: https://orcid.org/0000-0002-7767-1131

3 Instituto Nacional de Colonização e Reforma Agrária, Edifício Palácio do Desenvolvimento, Brasília, Brasil. marcelo.cunha@incra.gov.br

ORCID: https://orcid.org/0000-0001-7729-8093

4 Universidade Federal de Viçosa, Departamento de Engenharia Civil, Viçosa, Brasil. afonso.santos@ufv.br ORCID: https://orcid.org/0000-0001-7248-4524

Recebido: 03.2021 | Aceito: 04.2021

Resumo: No decorrer dos últimos anos têm ocorrido um significativo aumento de aplicações com Aeronaves Remotamente Pilotadas (RPAs) na área da Cartografia, o que torna necessário o conhecimento da qualidade obtida em tais aplicações. Diante disso, instituições de diversos países têm desenvolvido normas com a finalidade de avaliar a qualidade dos produtos geoespaciais. Além disso, desenvolveram também outras normas com a finalidade de englobar padrões de acurácia de posicionamento para ortoimagens digitais. Neste sentido, o objetivo deste artigo foi comparar três normas que abordam o controle de qualidade posicional aplicáveis à RPA: (i) ET-CQDG (Decreto $\mathrm{n}^{\circ}$ 89.817); (ii) a norma de Execução do INCRA n ${ }^{\circ} 2$ de 2018 e; (iii) a norma da ASP RS. No experimento prático, realizou-se o processo de avaliação da acurácia posicional de uma ortofoto obtida por RPA na região de Janaúba-MG. A ortofoto foi classificada na classe A para a escala de 1:1000 para a norma ET-CQDG. Já para a ASPRS ao nível de confiança de $95 \%$ o produto apresentou uma acurácia posicional de 0,428 metros e, de acordo com a norma do INCRA, os pontos obtidos por RPA podem ser utilizados para o georreferenciamento considerando todos tipos de limites. Através dos resultados obtidos, foi possível inferir que as normas necessitam de uma melhoria em suas metodologias, para que todo esse processo seja feito de forma clara e objetiva para o produtor e o usuário.

Palavras-chave: Acurácia posicional. ASPRS. RPA. INCRA. Decreto n 89.817/ET-CQDG.

Abstract: Over the past few years there has been a significant increase in applications with Remote Piloted Aircraft (RPAs) in the area of Cartography, which makes it necessary to know the quality obtained in such applications. Therefore, institutions from different countries have developed standards in order to assess the quality of geospatial products. In addition, they have also developed other standards with the purpose of encompassing positioning accuracy standards for digital orthoimages. In this sense, the purpose of this article was to compare three standards that address positional quality control applicable to RPA: (i) ET-CQDG (Decree No. 89,817); (ii) the INCRA Execution standard No. 2 of 2018 and; (iii) the ASP RS standard. In the practical experiment, the process of evaluating the positional accuracy of an orthofto obtained by RPA in the region of Janaúba-MG was carried out. The orthophoto was classified in class A for the scale of 1: 1000 for the standard ET-CQDG. For the ASPRS at the 95\% confidence level, the product had a positional accuracy of 0.428 meters and, according to the INCRA standard, the points obtained by RPA can be used for georeferencing considering all types of limits. Through the results obtained, it was possible to infer that the standards need an improvement in their methodologies, so that this whole process is done in a clear and objective way for the producer and the user.

Key-words: Positional accuracy. ASPRS. RPA INCRA. Decree No. 89.817/ET-CQDG. 


\section{INTRODUÇÃO}

A popularização das informações espaciais aumentou consideravelmente a demanda por dados com qualidade, atualizações mais frequentes e melhores resoluções espaciais. Assim, para suprir esta necessidade têm surgido novas tecnologias e métodos para mapear a superfície terrestre e gerar produtos cartográficos mais acurados (ALMEIDA et al., 2016).

Uma dessas tecnologias corresponde à utilização de Aeronaves Remotamente Pilotadas (RPA) para atividades de aerolevantamentos, que por sua vez, subsidiam as análises geoespaciais. No entanto, verificouse que os produtos oriundos de tal tecnologia permitiram estudos em delimitação de áreas de proteção permanente (APP) (CÂNDIDO; DA SILVA; FILHO, 2015), pesquisas em agricultura de precisão (RESENDE et al. 2020), análises de suscetibilidade à inundação (PONTES, et al. 2020), dentre outras aplicações.

Por ser uma tecnologia economicamente viável e de simples manuseio, houve um grande aumento da utilização de RPAs, tornando-se necessário o conhecimento da confiabilidade e acurácia dos seus produtos, principalmente na aplicação cartográfica. Maiores informações sobre trabalhos que englobam avaliação da acurácia posicional em produtos cartográficos podem ser encontradas em (SANTOS et al., 2016; FONSECA NETO et al. 2017; LISBOA et al. 2019).

Diante da necessidade de abordar a questão da qualidade em dados espaciais, diversos países e instituições elaboraram normas e padrões que englobam a análise da acurácia posicional de produtos cartográficos, como por exemplos os obtidos por RPAs. Em relação ao Brasil, a avaliação da acurácia posicional é realizada conforme as diretrizes do Decreto n 89.817 (BRASIL, 1984), aliado à Especificação Técnica para Controle de Qualidade de Dados Geoespaciais (ET-CQDG) de 2016, o qual realiza a classificação da qualidade posicional planimétrica e altimétrica em produtos cartográficos.

Além disso, no Brasil, foi elaborada a Norma de Execução nº 2 de 2018 do Instituto Nacional de Colonização e Reforma Agrária (INCRA) que estabelece os critérios para a avaliação da acurácia posicional em produtos gerados a partir de aerolevantamentos, para a utilização no processo de georreferenciamento de imóveis rurais.

Outra norma, reconhecida internacionalmente, é o padrão de qualidade da Associação de Fotogrametria e Sensoriamento Remoto dos Estados Unidos (ASPRS), que engloba padrões de acurácia de posicionamento para ortoimagem digital, dados planimétricos digitais, dados digitais de elevação e dados obtidos com LIDAR (Light Detection and Ranging) (HOLLER et al., 2018).

Dessa forma, devido à diversidade de normas existentes, surge a necessidade de análises e comparações entre estas. Nero (2005) analisou normas de diversos países referentes ao controle de qualidade posicional, sendo elaborada uma tabela comparativa, além de apresentar sugestões para incorporar na atualização da norma de controle posicional no Brasil.

Já Ariza e Atkinson (2008) analisaram diferentes metodologias presentes nas normas: National Map Accuracy Standard (NMAS) - EUA, STANAG 2215 - Standard from North Atlantic Treatment Organization (NATO), National Standard for Spatial Data Accuracy (NSSDA) - EUA, Accuracy Standards for Large Scale Maps (ASLSM) - EUA e MIL-STD-600001 - Department of Defense from United States (DoD) e verificaram que essa análise serve como um ponto de partida para o desenvolvimento de metodologias mais aprimoradas.

Pereira e Nero (2012) realizaram uma análise comparativa das normas da África do Sul, México, Austrália, Portugal, Japão e Brasil. Nesse estudo levaram em consideração o número de pontos de controle, o indicador estatístico, a aplicação (diferentes escalas), o erro padrão adotado, nível de confiança, tipo de análise com função normal uni ou bidimensional e observações gerais. A partir dos resultados encontrados, elaboraram sugestões para atualizar a norma vigente de controle posicional em Cartografia no Brasil, além de enfatizar as diferenças entre as normas.

Outro trabalho relevante é o de Zanetti et al. (2018), que realizaram uma comparação entre o Decreto $\mathrm{n}^{\circ}$ 89.817/ET-CQDG, a norma da ASPRS e a norma da STANAG 2.215 da Organização do Tratado do Atlântico Norte (OTAN). Os autores verificaram que dentre as normas avaliadas a STANAG é a mais bem estruturada e detalhada metodologicamente.

Carvalho e Silva (2018) abordaram uma revisão sobre os procedimentos metodológicos utilizados para a avaliação da qualidade posicional altimétrica no Brasil, além de realizar uma comparação com normas 
e artigos internacionais. Neste estudo, percebeu-se que pode ocorrer de alguns produtos geoespaciais serem classificados com padrões de qualidade não adequados, ao analisar variáveis que não apresentam distribuição normal. Devido a essas inconsistências, os autores sugerem evitar a utilização de dados não normais.

Saito et al. (2019) avaliaram as discrepâncias resultantes dos métodos Distância de Hausdorff e Influência do Vértice a partir do uso de feições lineares de hidrografia numérica e linhas de cumeada, extraídas de dois Modelos Digitais de Superfície. Neste estudo, os autores verificaram que as discrepâncias obtidas estão diretamente associadas ao desenvolvimento matemático dos métodos, além de ressaltarem a necessidade da existência de uma norma brasileira específica para a análise da acurácia posicional de um produto cartográfico utilizando feições lineares.

Mediante ao exposto e com base nos trabalhos já desenvolvidos, verifica-se que as normas são documentos que possuem padrões de qualidade para garantir um nível de confiabilidade, e padronizar o processo de produção cartográfica. Além disso, alguns dados de alta resolução são obtidos com RPAs que não possuem, por exemplo, câmeras com a calibração adequada em baixas altitudes, isso implica em dados que não possuem uma boa acurácia. Dessa forma, é fundamental a avaliação destes através da aplicação de normas que facilitem a análise e que forneça resultados acerca da sua qualidade posicional. É também importante que sejam realizadas análises e comparações entre as normas, deixando claro as diferenças em suas metodologias para que não haja dúvidas em suas interpretações e que seja feita a correta aplicação.

Assim, este artigo propõe analisar três normas que abordam o controle de qualidade posicional aplicáveis à RPA: (i) ET-CQDG (Decreto no 89.817); (ii) a norma de Execução do INCRA nº 2 de 2018 e; (iii) a norma da ASPRS. A escolha das normas utilizadas nesse estudo, tem a seguinte motivação: o Decreto $\mathrm{n}^{\circ}$ 89.817 aliado à ET-CQDG é a principal referência no Brasil na avaliação da qualidade posicional; a Norma de Execução do INCRA por ser uma nova alternativa a ser utilizada nesse processo visando produtos obtidos por aerofotogrametria, como por exemplo, utilizando RPAs; e a norma da ASPRS por apresentar procedimentos para avaliação de produtos obtidos por novas tecnologias. Sendo assim, este trabalho busca mostrar a aplicação de cada norma, bem como analisar o que poderia ser melhorado para uma análise mais eficiente.

Portanto, o objetivo principal é realizar uma análise comparativa das normas, aplicando-as na avaliação da acurácia posicional planimétrica de um produto cartográfico obtido por RPA. Especificamente, buscou-se ao realizar essa análise, avaliar a qualidade posicional de uma ortofoto em um assentamento do INCRA em Janaúba, Minas Gerais. Posteriormente, com o resultado obtido, foi explicitado o que cada norma engloba, bem como os aspectos ausentes.

\section{NORMAS PARA AVALIAÇÃO DA ACURÁCIA POSICIONAL}

\section{$2.1 \quad$ ET-CQDG}

A ET-CQDG é uma norma baseada na ISO (2013) que foi publicada pela DSG em 2016, e que apresenta uma metodologia para avaliar a acurácia posicional de dados cartográficos de acordo com o Decreto $n^{\circ} 89.817$ aliado à ET-ADGV. Esta norma tem como base a utilização de feições pontuais, onde é comparado a posição dos dados de teste em relação aos dados de referência.

Além disso, a ET-CQDG utiliza os conceitos de amostragem conforme a ISO (1999) (inspeção lote a lote) e ISO (1985). De acordo com a ISO (1999), o plano de amostragem é a combinação entre o tamanho da amostra (n) e o critério de aceitação (Ac). O tamanho de amostra é a quantidade de itens em um lote que será inspecionado, e o critério de aceitação é a quantidade máxima de não conformidade com um determinado requisito.

Para determinar o tamanho amostral, o produto a ser avaliado deve ser particionado em células de $4 \mathrm{x}$ $4 \mathrm{~cm}$ na escala a ser testada. Sendo assim, em um produto 1:10.000 a célula teria $400 \mathrm{x} 400 \mathrm{~m}$, já em um produto com escala 1:25.000 a célula possuiria 1000 x $1000 \mathrm{~m}$. Após a divisão do produto a ser avaliado em células, o avaliador deve verificar cada célula para analisar se esta é válida para compor a população que será avaliada no lote. São consideradas células válidas apenas aquelas que contenham feições onde seja possível identificar um ponto de checagem no terreno.

Considerando a inspeção lote a lote, após fixar o Limite de Qualidade Aceitável (LQA) e o tamanho 
da população para cada procedimento, o tamanho da amostra é obtido de acordo com a Tabela 44 e a Tabela 45 no Anexo A da norma ET-CQDG. Com o tamanho do lote (população) e o nível de inspeção (normalmente utilizado é o nível II), determina-se a letra código para o tamanho da amostra usando a Tabela 44. Posteriormente utiliza-se a Tabela 45 através da letra código e do LQA para determinar o tamanho da amostra (n) e o número de aceitação (Ac). Se o tamanho de amostra não possuir o número de aceitação para essa letra, o valor correspondente de $\mathrm{n}$ e Ac deve ser adotado.

Já considerando a inspeção por lote isolado, as taxas de erro aceitável nesta especificação são expressas como LQA, ou seja, podem ser aplicadas para os planos de amostragem lote a lote da ISO (1999). Entretanto, a ISO (1985) indexa seus planos de amostragem pela qualidade limite (QL), sendo necessário fazer a conversão do LQA para QL visando a aplicação do procedimento de amostragem a produtos isolados. Vale ressaltar que a QL é sempre menor que o LQA, contudo, a diferença diminui com o aumento do tamanho da amostra (ISO 2859-0:1995). Ressalta-se que a Tabela 46 da norma apresenta os valores definidos nesta especificação para converter de LQA para QL.

Através do LQA e o tamanho da população, verifica-se na Tabela 46 qual o QL correspondente, para que seja possível determinar o tamanho da amostra (n) e o número de aceitação (Ac) contido na Tabela 47. Caso o tamanho da população não possua $n$ e Ac para esse QL, o valor correspondente de $n$ e Ac deve ser adotado.

Diante disso, para que um produto seja acurado posicionalmente em uma determinada escala, é necessário atender dois requisitos:

1) $90 \%$ das discrepâncias (Eq. (1)) testadas devem ser menores ou iguais ao PEC-PCD para a classe e escala testada;

2) O RMS (Eq. (2)) da amostra de discrepâncias testadas deve ser menor ou igual EP para a classe e escala testada.

Onde:

$$
\begin{gathered}
d_{p j}=\sqrt{\left(X_{T}-X_{R}\right)^{2}+\left(Y_{T}-Y_{R}\right)^{2}} \\
R M S=\sqrt{\frac{\sum_{j=1}^{n}(d p j)^{2}}{n-1}}
\end{gathered}
$$

Sendo:

$\mathrm{d}_{\mathrm{p} j}$ : discrepância posicional da feição j;

n: número de feições de checagem;

$\mathrm{X}_{\mathrm{T}}, \mathrm{Y}_{\mathrm{T}}$ : coordenadas do ponto de teste (checagem);

$\mathrm{X}_{\mathrm{R}}, \mathrm{Y}_{\mathrm{R}}$ : coordenadas do ponto de referência.

Dessa forma, a Tabela 1 apresenta as tolerâncias do PEC e PEC-PCD utilizadas na avaliação da acurácia posicional planimétricas baseadas no padrão descrito na ET-CQDG (DSG, 2016), utilizando como base o Decreto ${ }^{\circ} 89.817$ (BRASIL, 1984).

Tabela 1 - Tolerâncias para avaliação da acurácia posicional planimétrica de acordo com o Decreto n 89.817/ ET-

ADGV.

\begin{tabular}{c|c|c|c}
\hline \multirow{2}{*}{ Classe PEC } & Classe PEC-PCD & Planimetria \\
\cline { 3 - 4 } & & PEC (mm) & EP $(\mathbf{m m})$ \\
\hline- & A & 0,28 & 0,17 \\
A & B & 0,5 & 0,3 \\
B & C & 0,8 & 0,5 \\
C & D & 1,0 & 0,6 \\
\hline
\end{tabular}

Fonte: Adaptada de Santos (2010).

É importante ressaltar que a ET-CQDG detalha a metodologia a ser feita para a amostragem em produtos cartográficos digitais, contudo não contém em sua especificação nada relacionado a testes para verificar a tendência e detecção de outliers. Além disso, Santos et al. (2016) afirmam que a ET-CQDG possui alguns erros de definição como, por exemplo, o uso inicial do termo "Padrão de Exatidão Cartográfica (PEC), 
posteriormente utiliza Erro Máximo (EM) e finaliza utilizando o Erro Médio.

\subsection{Norma da ASPRS (2014)}

Segundo Zanetti et al., (2018), o padrão da ASPRS refere-se ao estado atual da tecnologia de mapeamento. Por esse motivo, as normas de acurácia da ASPRS foram desenvolvidas para os Padrões de Acurácia para Dados Digitais Geoespaciais em 2014.

Esse padrão inclui normas de acurácia posicional para ortoimagens, dados planimétricos e dados digitais de elevação. As classes de acurácia são baseadas nos valores do RMSE (Raiz Quadrada do Erro Médio) e foram revisadas e atualizadas a partir do padrão de 1990 para abordar as maiores precisões possíveis com as novas tecnologias (ASPRS, 2014). No entanto, esse padrão trata da acurácia na localização geográfica de produtos geoespaciais e não se destina a cobrir a precisão da classificação de mapas temáticos. Além disso, o padrão não especifica as melhores práticas ou metodologias necessárias para atender aos limites de precisão estabelecidos (ASPRS, 2014).

Portanto, o objetivo geral da norma ASPRS 2014 é substituir os padrões existentes para refletir melhor as novas geotecnologias, para ser utilizada por fornecedores e usuários de dados geoespaciais, com a finalidade de determinar os requisitos de acurácia para o posicionamento e assim relatar a precisão dos dados (ZANETTI et al., 2018).

É importante salientar que a ASPRS 2014 especifica a quantidade de pontos de checagem a ser utilizada na avaliação do produto cartográfico de acordo com a área de estudo, conforme a Tabela 2 (Zanetti et al., 2018). Porém, a mesma não menciona testes para detecção de tendência e outliers. No entanto, ao se aplicar o controle de qualidade é de suma relevância realizar a análise das tendências e verificar a presença de outliers, uma vez que um dado tendencioso inclui erros sistemáticos e impossibilita sua integração.

Tabela 2 - Recomendação da ASPRS 2014 para o número de pontos de checagem de acordo com a área de estudo.

\begin{tabular}{c|c}
\hline Tamanho da Área $\left(\mathbf{K m}^{\mathbf{2}}\right)$ & Número total de amostra (Planimétrica) \\
\hline 5000 & 20 \\
$501-750$ & 25 \\
$751-1000$ & 30 \\
$1001-1250$ & 35 \\
$1251-1500$ & 40 \\
$1501-1750$ & 45 \\
$1751-2000$ & 50 \\
$2001-2250$ & 55 \\
$2251-2500$ & 60 \\
\hline
\end{tabular}

Fonte: Adaptada da ASPRS (2014).

Conforme Zanetti et al. (2018), os padrões anteriores a norma ASPRS 2010 utilizavam classes de acurácia (Classe 1, Classe 2 e Classe 3) diretamente relacionados a escala de mapeamento, como no Decretolei $n^{\circ}$ 89.817. Porém, a ASPRS 2014 propõe uma classificação baseada no RMS do produto. Sendo assim, após calcular as discrepâncias posicionais dos pontos de checagem calculam-se seus valores de RMS para as componentes x e y (Eq. (3) e Eq. (4)).

$$
\begin{aligned}
& R M S E_{x}=\sqrt{\frac{1}{n} \sum_{i=1}^{n}\left(x_{\text {test }}-x_{\text {ref }}\right)^{2}} \\
& R M S E_{y}=\sqrt{\frac{1}{n} \sum_{i=1}^{n}\left(y_{\text {test }}-y_{\text {ref }}\right)^{2}}
\end{aligned}
$$


Onde:

$x_{\text {test }}:$ Valor da abcissa no produto teste;

$x_{r e f}$ : Valor da abcissa no produto de referência;

$y_{\text {test }}$ : Valor da ordenada no produto teste;

$y_{\text {ref }}$ : Valor da ordenada no produto de referência.

Após o cálculo do RMSE das componentes x e y do produto é feita uma comparação destes valores com a especificação do produto a ser avaliado, conforme a Tabela 3. O padrão da ASPRS permite obter a qualidade e dependendo do projeto usar uma tolerância para a variável X, conforme explicitado na Tabela 4 (ASPRS, 2014).

Tabela 3 - Padrão de acurácia horizontal de acordo com a ASPRS (2014).

\begin{tabular}{c|c|c|c}
\hline \multirow{2}{*}{ Classe Acurácia Horizontal } & \multicolumn{3}{|c}{ Acurácia Absoluta } \\
\cline { 2 - 4 } & RMSEx e RMSEy & RMSEr & Nível de Confiança 95\% \\
\hline $\mathrm{X}$ & $\leq \mathrm{X}$ & $\leq 1,414 * \mathrm{X}$ & $\leq 2,448 * \mathrm{X}$ \\
\hline
\end{tabular}

Fonte: ASPRS (2014).

Tabela 4 - Classes comuns de acurácia horizontal para ortofotos digitais e padrões horizontais de acordo com a ASPRS (2014).

\begin{tabular}{c|c|c}
\hline $\begin{array}{c}\text { Classe de acurácia horizontal [RMSEx e } \\
\text { RMSEy }(\mathbf{c m})]\end{array}$ & RMSEr $(\mathbf{c m})$ & $\begin{array}{c}\text { Acurácia horizontal com nível de confiança de } \\
\mathbf{9 5 \%}\end{array}$ \\
\hline 0,63 & 0,9 & 1,5 \\
1,25 & 1,8 & 3,1 \\
2,5 & 3,5 & 6,1 \\
5 & 7,1 & 12,2 \\
7,5 & 10,6 & 18,4 \\
10 & 14,1 & 24,5 \\
12,5 & 17,7 & 30,6 \\
15 & 21,2 & 36,7 \\
17,5 & 28,3 & 42,8 \\
20 & 31,8 & 49 \\
\hline
\end{tabular}

Fonte: Adaptado da ASPRS (2014).

Ao avaliar a qualidade cartográfica em ortofotos, a norma da ASPRS também inclui recomendações para o uso dos valores RMSEx e RMSEy encontrados. A norma também inclui o princípio geral de determinar o uso correto da ortoimagem a partir de três níveis diferentes de precisão de posicionamento. Os valores listados como "Trabalhos com maior acurácia" indicam RMSEx e RMSEy em 1 pixel devido às resoluções mais altas das tecnologias atuais. Esta classe é adequada se a acurácia espacial for de grande importância. Os valores listados como "Mapeamento e operação GIS padrão" especificam as classes de precisão de 2 pixels de RMSEx e RMSEy. Essa precisão é adequada para aplicações padrão de alta qualidade. Uma precisão de RMSEx e RMSEy de 3 ou mais pixels seria considerada adequado para "navegação e trabalhos menos precisos" quando não é necessária maior acurácia (Zanetti et al., 2018).

\subsection{Norma de Execução $n^{\circ} 2$ do INCRA}

Em 19 de fevereiro de 2018, foi regulamentada a Norma de Execução do INCRA n 2, que estabelece critérios para a aplicação e avaliação de produtos gerados a partir de aerofotogrametria para determinação de vértices definidores de limites de imóveis rurais em atendimento ao parágrafo $3^{\circ}$ do artigo 176 da Lei $n^{\circ} 6.015$.

Esta norma estabelece que para a devida avaliação da acurácia posicional, deve-se seguir alguns critérios que estão descritos abaixo (INCRA, 2018):

- Utilização de no mínimo 20 pontos de checagem, sendo estes bem identificados em campo;

- Teste estatístico que comprove a normalidade das discrepâncias posicionais planimétricas ao nível de confiança de $95 \%$, utilizando o método de Shapiro-Wilk;

- Teste de tendência ao nível de $90 \%$, utilizando-se o teste t de Student, que comprove a não 
tendenciosidade; e

- $100 \%$ das discrepâncias posicionais serem menores ou iguais à precisão posicional correspondente a cada tipo de limite.

Entretanto, a norma do INCRA não especifica a dimensão da área a serem coletados os pontos de checagem, apenas diz que devem estar bem identificados em campo. Além disso, os dados necessitam seguir a distribuição normal para que seja possível utilizar o teste t de Student para verificar se há tendência. A normalidade dos dados é verificada pelo teste Shapiro-Wilk, conforme é mostrado em Santos et al. (2016).

Já o teste $\mathrm{t}$ de Student para analisar tendência em produtos cartográficos, foi proposto por Merchant (1982), e é recomendado utilizá-lo para amostras menores que 30, acima desse valor deve-se adotar o teste Z.

Como pode ser observado, essa norma mostra que deve ser feita a análise de tendência, e por considerar que $100 \%$ das discrepâncias deve ser menor que o limite adotado, não pode haver outliers, o que torna mais rigoroso o processo de obtenção dos pontos de checagem.

\section{MATERIAIS E MÉTODOS}

Ao longo dos tópicos seguintes, serão apresentados o delineamento da área de estudo onde a pesquisa foi executada, os materiais, e a metodologia proposta para comparar a aplicação das normas em RPA.

\section{1 Área de estudo}

A área de estudo (Figura 1) está localizada no município de Janaúba no estado de Minas Gerais. A região possui 780 hectares aproximadamente.

Figura 1 - Mapa de localização da área de estudo.

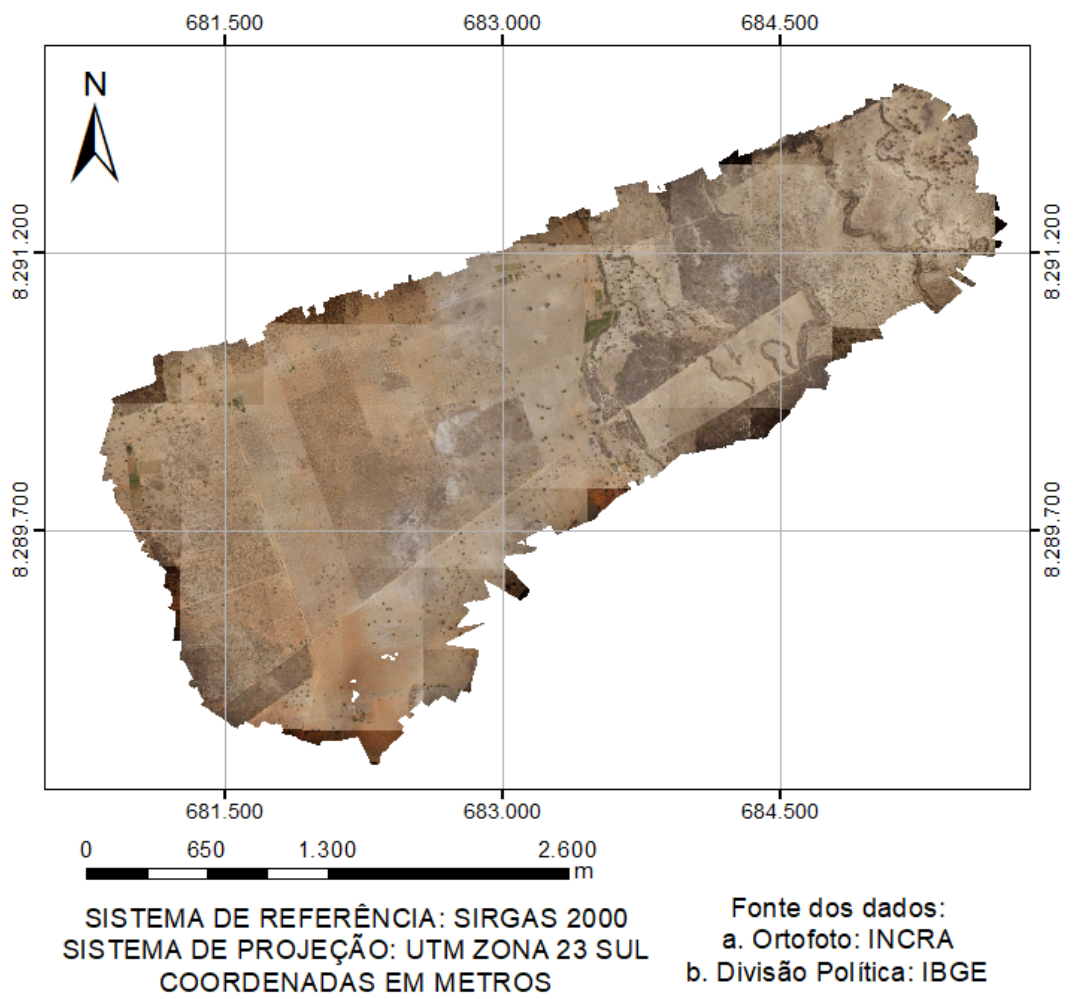

Fonte: Os autores (2021).

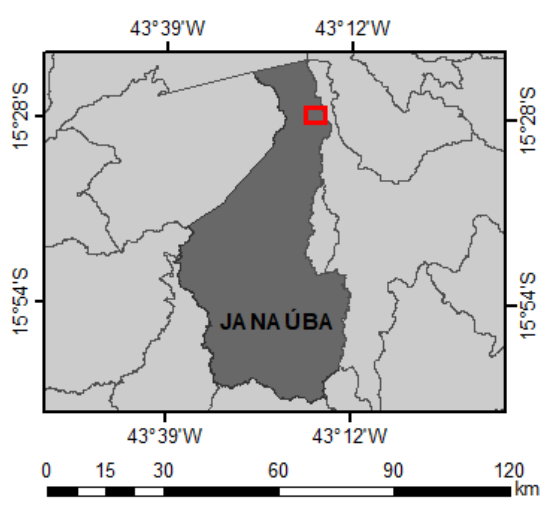

Sistema de Projeção: Plate Carrêe Sistema de Referência: SIRGAS 2000

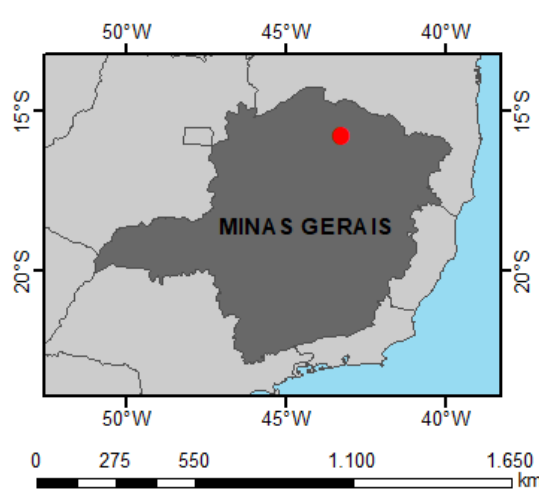

Sistema de Projeção: Plate Carrêe Sistema de Referência: SIRGAS 2000 


\subsection{Materiais utilizados}

A região de estudo foi levantada através de um levantamento aerofotogramétrico, através do equipamento VANT Echar 20C de propriedade do INCRA, no dia 26 de outubro de 2017 com uma altitude de voo de aproximadamente $310 \mathrm{~m}$.

Foi utilizado o software fotogramétrico Agisoft PhotoScan 1.3.2 para o processamento das imagens. A ortofoto resultante do processamento tem resolução espacial de $4,89 \mathrm{~cm}$.

Os pontos de checagem foram coletados em campo através de um receptor modelo Hiper GR5 de dupla frequência (L1/L2), utilizando o posicionamento relativo estático. O sistema de referência utilizado neste trabalho foi o SIRGAS 2000, com coordenadas plano retangulares estimadas no sistema de projeção UTM, no fuso $23 \mathrm{~S}$.

Além disso, também foi utilizado o software QGIS versão 3.4.11 para a coleta dos pontos homólogos na ortofoto e o software GeoPEC para a avaliação da acurácia posicional a partir da norma brasileira (GEOPEC, 2021). A planilha eletrônica Excel foi utilizada para os cálculos das normas da ASPRS e INCRA.

\subsection{Metodologia}

Os fluxogramas abaixo explicitam os principais procedimentos utilizados ao longo desse trabalho, com a finalidade de realizar a avaliação da acurácia posicional de acordo com a metodologia proposta por cada norma. A Figura 2 ilustra um esquema com os principais critérios exigidos pela ET-CQDG (2016) para avaliação da acurácia posicional em produtos cartográficos.

Figura 2 - Fluxograma metodológico das recomendações inerentes à avaliação da acurácia posicional de acordo com a ET-CQDG (2016).

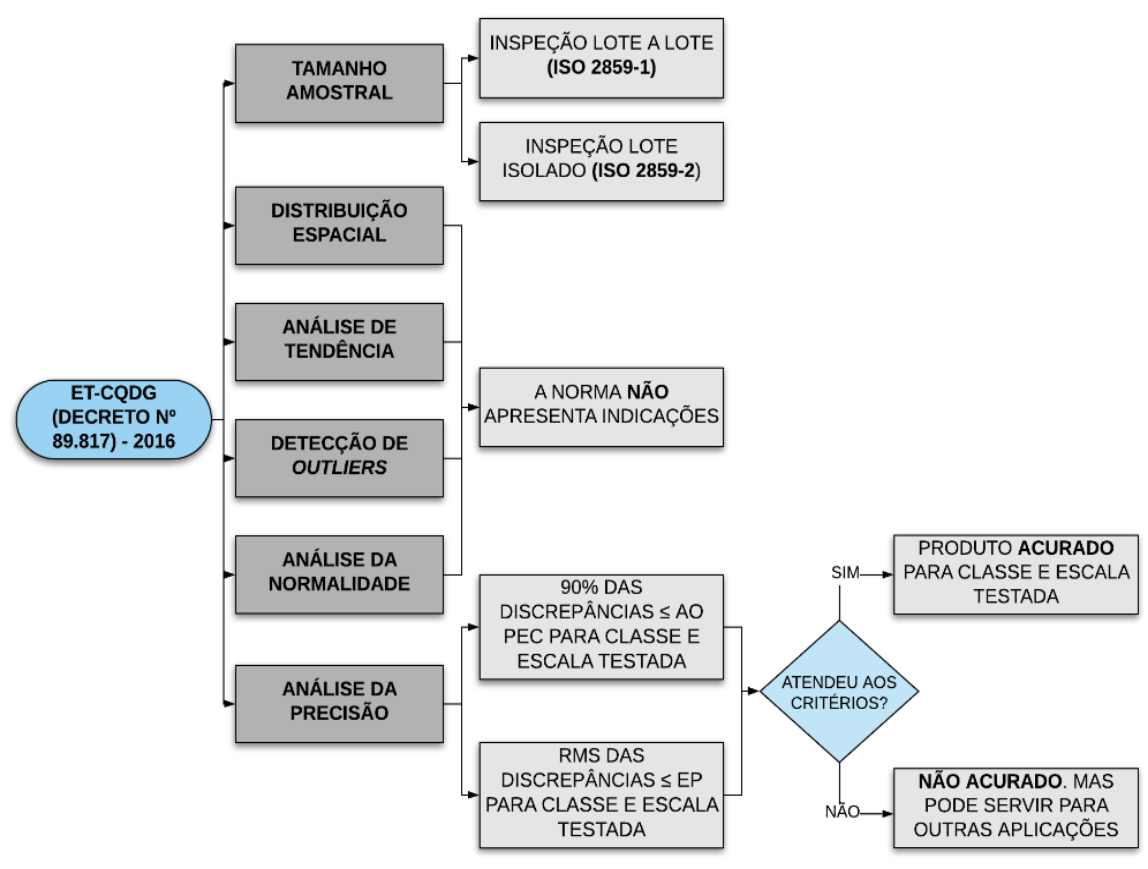

Fonte: Os autores (2021).

O fluxograma abaixo detalha as recomendações sugeridas pela norma americana (ASPRS, 2014) para avaliação da acurácia posicional. 
Figura 3 - Fluxograma metodológico das recomendações inerentes à avaliação da acurácia posicional de acordo com a ASPRS (2014).

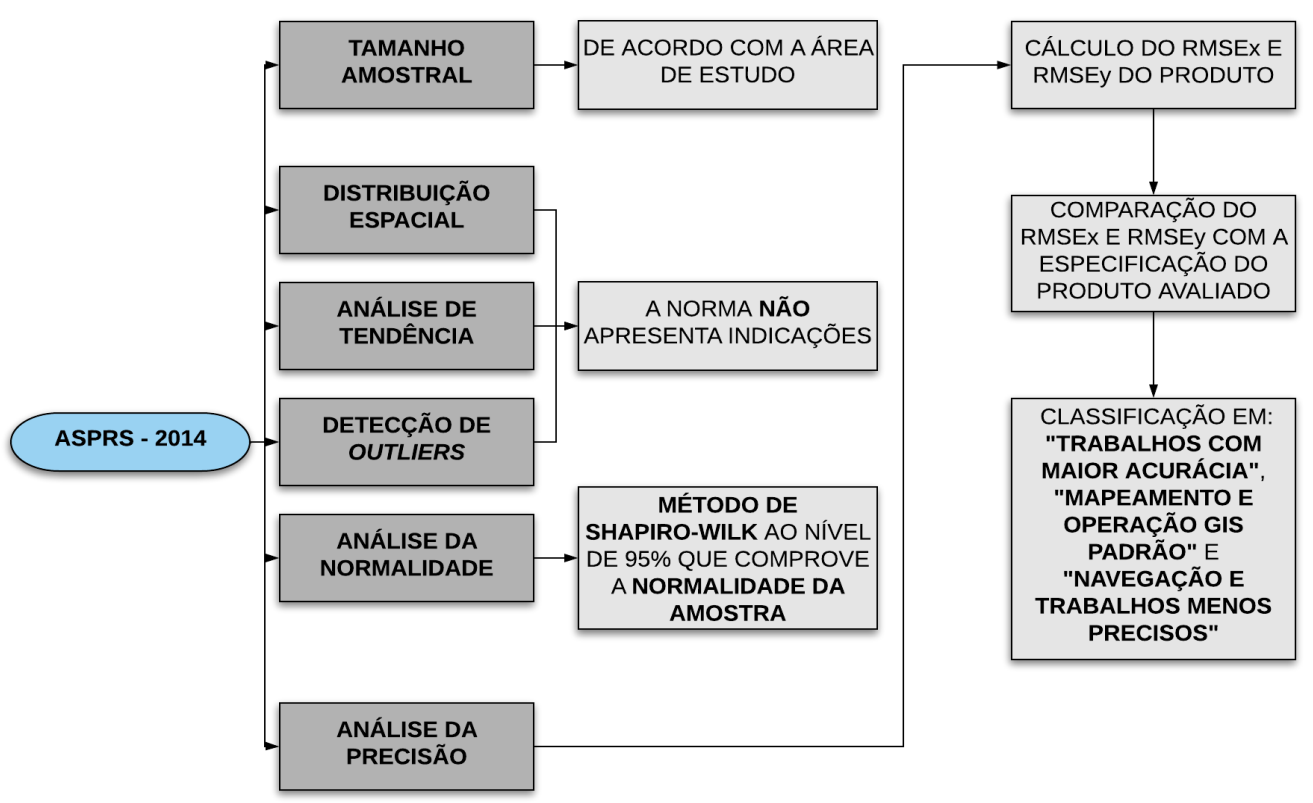

Fonte: Os pelos autores (2021).

No Brasil além das recomendações da ET-CQDG (2016), o Instituto Nacional de Colonização e Reforma Agrária (INCRA) também sugere diretrizes para avaliação da acurácia posicional em produtos cartográficos oriundos de levantamentos com RPA. Desta forma, a Figura 4 ilustra o fluxograma com as recomendações da norma do INCRA (2018).

Figura 4 - Fluxograma metodológico das recomendações inerentes à avaliação da acurácia posicional de acordo com o INCRA (2018).

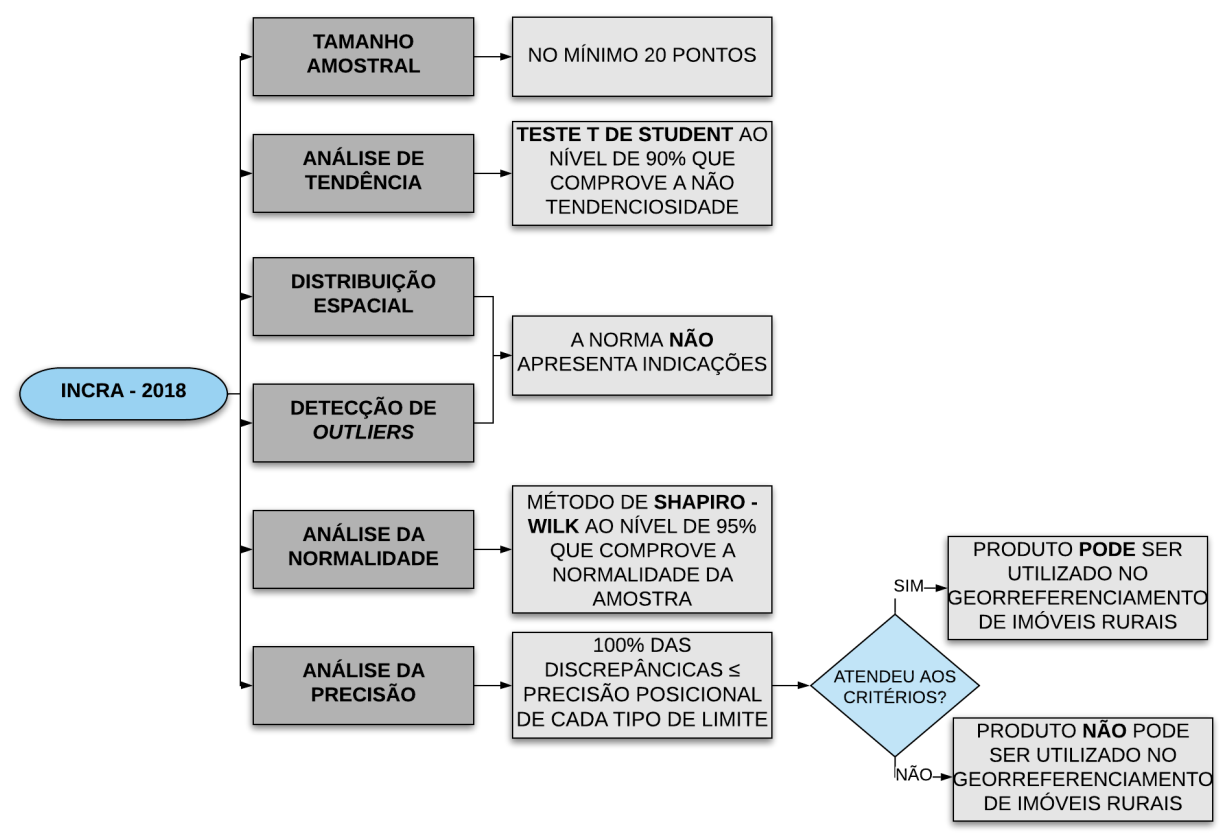

Fonte: Os autores (2021).

Ao longo deste trabalho, foi calculado o tamanho amostral dos pontos de checagem na ortofoto, mediante aos padrões estabelecidos pela ET-CQDG nas normas ISO (1999) e ISSO (1985) para um produto lote isolado.

Posteriormente, foi executada a análise estatísticas e de classificação da ET-CQDG e da norma do 
INCRA com o software GeoPEC. Por fim, foi realizada a comparação da aplicação em RPA com as normas da ET-CQDG, ASPRS e INCRA, com o objetivo de verificar qual norma possui uma metodologia estatística mais completa e uma classificação mais restritiva.

\section{RESULTADOS E ANÁLISES}

Visando a definição da distribuição das amostras, a área de estudo foi dividida em 13.365 quadrantes de 4x4 cm de lado na escala 1:1.000 conforme a metodologia da ET-CQDG. Como a ASPRS determina vinte pontos considerando o tamanho da área de estudo em questão, e a normativa do INCRA também determina o mínimo de 20 pontos de checagem, foram utilizados vinte pontos para analisar os resultados pelas três metodologias, conforme a Figura 5. Os pontos de checagem foram escolhidos de forma bem distribuídas pela região. Para confirmar este processo, foi aplicado a Função K de Ripley para analisar o padrão de distribuição espacial, conforme Santos et al. (2016). Obteve o padrão disperso como resultado, indicando uma boa distribuição dos pontos de checagem na região estudada.

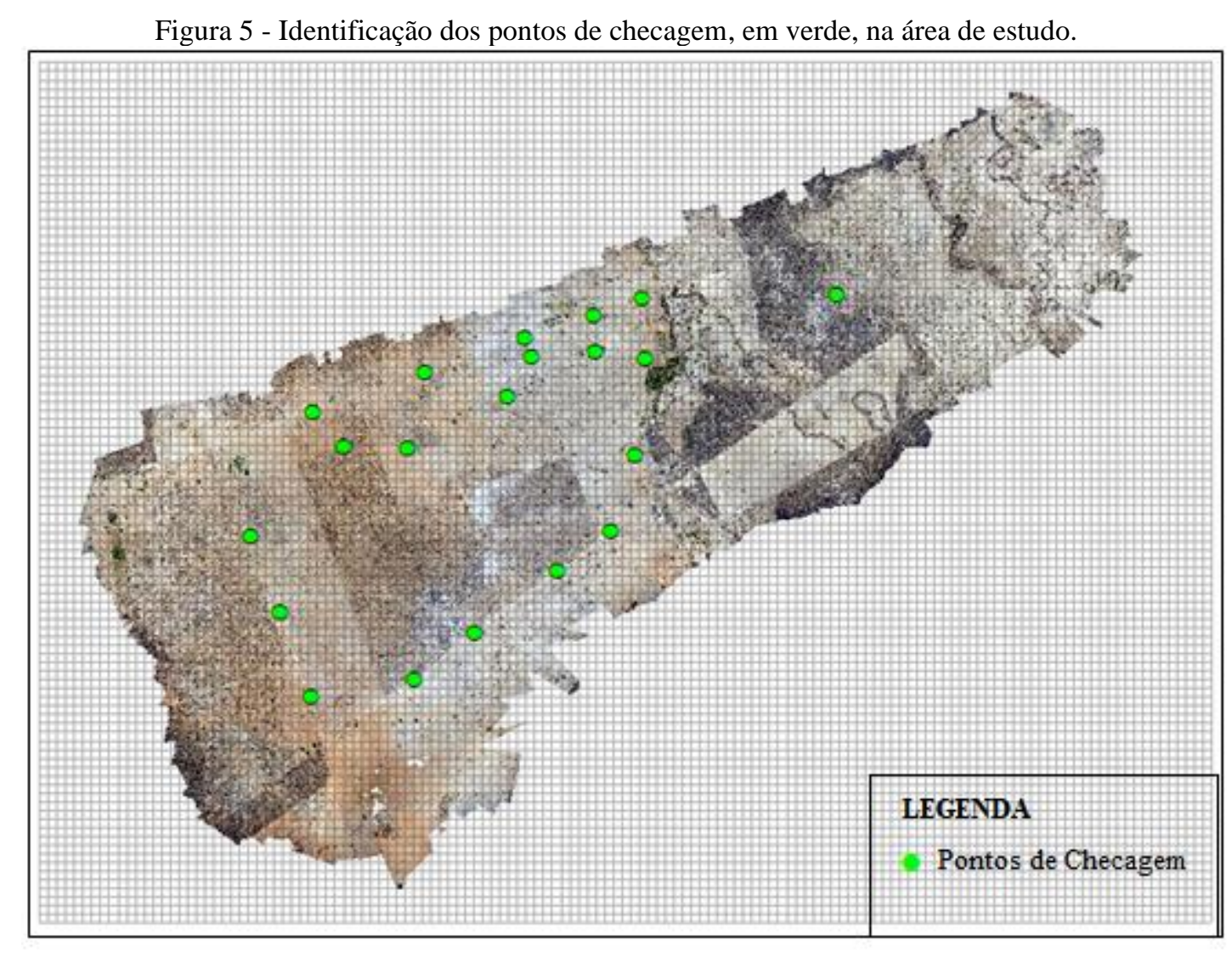

Fonte: Os autores (2021).

De posse das discrepâncias posicionais planimétricas de cada ponto de checagem, que é calculada conforme a equação 1, obteve-se as estatísticas descritivas da amostra. Dessa forma o valor do RMS foi igual a 15,8 cm, a média de $13,8 \mathrm{~cm}$ e desvio padrão de $7,8 \mathrm{~cm}$, como pode ser visto na Tabela 5. 
Tabela 5 - Estatísticas descritivas para uma amostra de 20 pontos.

\begin{tabular}{c|c|c|c}
\hline Estatística & Este & Norte & Posicional \\
\hline média (m) & 0,026 & 0,037 & 0,138 \\
desvio (m) & 0,099 & 0,119 & 0,079 \\
variância (m²) & 0,010 & 0,014 & 0,006 \\
RMS (m) & 0,101 & 0,122 & 0,288 \\
máximo (m) & 0,232 & 0,230 & 0,018 \\
mínimo (m) & $-0,174$ & $-0,202$ & 0,118 \\
soma (m) & 0,188 & 0,269 & \\
\hline
\end{tabular}

Fonte: Os autores (2021).

A norma de execução do INCRA exige que a análise de tendência seja feita por meio do teste estatístico $\mathrm{t}$ de Student, ao nível de confiança de $90 \%$. Esse teste exige que a amostra siga a distribuição normal (SANTOS et al. 2016), dessa forma essa norma recomenda utilizar o método de Shapiro-Wilk (SANTOS et al. 2016) a $95 \%$ de confiança, bem como a norma ASPRS. Através da aplicação deste método foi constatada a normalidade dos dados, de acordo com a Tabela 6.

Tabela 6 - Resultado do teste de normalidade pelo Shapiro-Wilk.

\begin{tabular}{c|c|c|c|c}
\hline Wcal (E) & Wcal (N) & p-value E & p-value $\mathbf{~}$ & Resultado \\
\hline 0,984 & 0,963 & 0,971 & 0,609 & Amostra normal \\
\hline \multicolumn{4}{c}{ Fonte: Os autores (2021). }
\end{tabular}

Os resultados da análise de tendência pelo t de Student, tanto na componente Este quanto na componente Norte, encontram-se na Tabela 7. De acordo com esses resultados, verifica-se que não há efeitos sistemáticos nos dados, ou seja, não há tendência.

Tabela 7 - Teste de tendência pelo t de Student.

\begin{tabular}{c|c|c|c}
\hline Coord & t tab & t cal & Resultado \\
\hline $\operatorname{di}(\mathrm{E})$ & 1,729 & 1,192 & Não existe efeito sistemático \\
$\operatorname{di}(\mathrm{N})$ & 1,729 & 1,421 & Não existe efeito sistemático \\
\hline
\end{tabular}

Fonte: Os autores (2021).

Para uma visualização melhor das discrepâncias em relação à coordenada Este e Norte, foi gerado o gráfico das discrepâncias (Figura 7).

Figura 7 - Gráfico das discrepâncias.

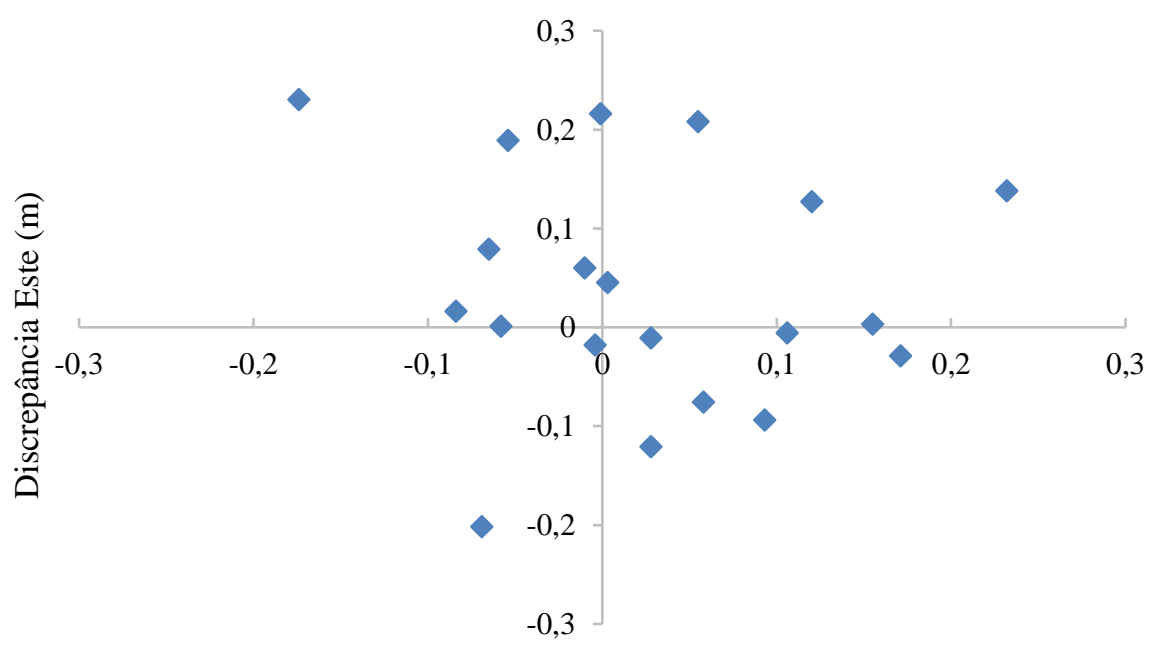

Discrepância Norte (m)

Fonte: Os autores (2021). 
Considerando as tolerâncias do Decreto 89.817/ET-CQDG, a ortofoto foi classificada na classe A com a classe 1:1.000 de acordo com a Tabela 8. Obteve-se essa classificação, pois o RMS de 0,158 m foi menor que o EP de 0,17 m dessa classe, e as discrepâncias da amostra foram 100\% menores que o valor do PEC de $0,28 \mathrm{~m}$.

Tabela 8 - Classificação do produto.

\begin{tabular}{c|c|c|c|c|c|c}
\hline Classe PEC & $\begin{array}{c}\text { Classe PEC- } \\
\text { PCD }\end{array}$ & PEC $(\mathbf{m})$ & EP $(\mathbf{m})$ & $\begin{array}{c}\text { \%di(abs) } \\
\text { PEC }\end{array}$ & RMS < PEC & Resultado \\
\hline- & A & 0,28 & 0,17 & 100 & Não & Aprovado \\
A & B & 0,50 & 0,30 & 100 & Sim & Aprovado \\
B & C & 0,80 & 0,50 & 100 & Sim & Aprovado \\
C & D & 1 & 0,60 & 100 & Sim & Aprovado \\
\hline
\end{tabular}

Fonte: Os autores (2021).

Dessa forma, de acordo com as condições do Decreto 89.817/ET-CQDG, o produto é acurado posicionalmente, já que não apresentou tendência e foi classificado na classe A.

Em relação à norma do INCRA, 100\% das discrepâncias posicionais devem ser menores ou iguais à precisão posicional do limite utilizado. Nesse caso, considerando limites artificiais a tolerância é de $50 \mathrm{~cm}$ conforme o Manual Técnico de Posicionamento do INCRA de 2013. Já em relação aos limites naturais e inacessíveis, a tolerância é de 3,0 m e 7,5 m respectivamente. Sendo assim, considerando as discrepâncias de cada ponto, verifica-se que esse requisito foi atendido para todos os tipos de limites, já que a discrepância máxima foi de $28,8 \mathrm{~cm}$.

Já a norma da ASPRS não possui padrões de acurácia com base nos valores do RMS. Nesse estudo, o valor do RMSEx foi de 0,101 m e RMSEy de 0,122 $\mathrm{m}$. Analisando a lista de classes comuns da ASPRS, a ortofoto possui a acurácia horizontal na classe RMSEx ou RMSEy de 0,175 m. De acordo com esse padrão, como a resolução da ortofoto é de 4,89 cm e considerando os valores de RMSEx e RMSEy obtidos, a ortofoto pode ser utilizada para "trabalhos com maior acurácia", visto que o RMSEx e RMSEy se enquadram em 1 pixel. Ao nível de confiança de $95 \%$, o produto apresenta uma acurácia posicional absoluta de 0,428 metros.

Em resumo, tem-se os resultados na Tabela 9.

\begin{tabular}{c|c|c|c}
\multicolumn{4}{c}{ Tabela 9 - Resultados obtidos aplicando as três normas na área de estudo. } \\
\hline Normas & ET-CQDG & INCRA & ASPRS \\
Resultado & $\begin{array}{c}\text { Escala 1: } 1.000 \\
\text { Classe A }\end{array}$ & $\begin{array}{c}\text { Pontos obtidos por RPA podem } \\
\text { ser utilizados para } \\
\text { georreferenciamento }\end{array}$ & $0,428 \mathrm{~m}$ \\
\hline
\end{tabular}

Fonte: Os autores (2021).

Considerando o padrão de acurácia posicional brasileiro (Decreto ${ }^{\circ}$ 89.817/ET-CQDG), verifica-se que apesar de ser necessário verificar o tamanho da amostra a ser utilizada, conforme os procedimentos da ISO 2.858, o mesmo não aborda como deve ser feita a análise da distribuição espacial de toda a área em estudo, sendo este um fator importante a ser considerado na avaliação da acurácia posicional de dados espaciais, conforme é dito em Santos et al. (2016).

Além disso, a norma do INCRA apenas afirma que a amostra deve estar bem distribuída pela área de estudo. Vale ressaltar que de acordo com Zanetti et al. (2018), a ET-CQDG define a fórmula do RMS, mas utiliza o termo "Erro Médio Quadrático".

Em relação à norma de execução $\mathrm{n}^{\circ} 02$ do INCRA, esta determina o número mínimo de pontos de checagem que devem ser utilizados, mas não especifica nada relacionado ao tamanho da área de estudo. Sendo assim, um questionamento que pode surgir é se avaliar uma área com 20 pontos e posteriormente fazer a mesma análise dessa área em sub-regiões, será que o resultado seria compatível?

Entretanto vale ressaltar que dentre as normas adotadas, é a única que deixa explícito como deve ser feita a verificação da tendência e qual teste utilizar para a análise de normalidade. Ademais, essa norma é mais restritiva que o Decreto ${ }^{\circ}$ 89.817/ET-CQDG, pois 100\% das discrepâncias devem ser menores que a tolerância do limite utilizado, e já o decreto exige que seja no mínimo $90 \%$ menor ou igual que o valor do PEC para a 
classe e escala testada.

Já a norma da ASPRS apenas calcula a acurácia do produto com base no RMS posicional ao nível de confiança de 95\%, não classificando o produto analisado de acordo com alguma escala (ZANETTI et al., 2018). Esta norma adota o tamanho amostral com base no tamanho da área, mas não faz a detecção de outliers.

Em resumo, a adoção dessas normas possibilitou verificar suas diferenças e a importância de se analisar alguns aspectos com indicadores estatísticos que é analisada por alguma, mas não por outra. A seguir, através do Quadro 1 serão apresentados uma visão global dos diferentes aspectos entre as normas avaliadas neste estudo.

Quadro 1 - Resumo dos aspectos revisados das metodologias de avaliação da acurácia posicional das normas analisadas.

\begin{tabular}{|c|c|c|c|}
\hline Nome da Norma & ASPRS & $\begin{array}{c}\text { Decreto }^{\circ} \text { 89.817/ET- } \\
\text { CQDG }\end{array}$ & INCRA \\
\hline Ano de publicação & 2014 & 2016 & 2018 \\
\hline Indicação de escala & - & - & - \\
\hline $\begin{array}{l}\text { Indicação do tipo de elemento ao qual } \\
\text { a avaliação é aplicada }\end{array}$ & $\begin{array}{c}\text { Mapas, ortofotos, Lidar, e } \\
\text { dados geoespaciais de } \\
\text { forma geral }\end{array}$ & Mapas & $\begin{array}{c}\text { Produtos cartográficos } \\
\text { elaborados a partir de } \\
\text { aerofotogrametria por (RPA) }\end{array}$ \\
\hline Tamanho amostral & De acordo com a área & $\begin{array}{c}\text { De acordo com a área e } \\
\text { baseada na ISO } 2859\end{array}$ & No mínimo 20 pontos \\
\hline Distribuição espacial & Não & Não & Não \\
\hline Precisão do Produto de Referência & - & $3 \mathrm{x}$ mais precisa & - \\
\hline $\begin{array}{c}\text { Outliers: se há uma indicação relativa } \\
\text { à sua eliminação ou como lidar com } \\
\text { eles }\end{array}$ & Não & Não & Não \\
\hline $\begin{array}{l}\text { Tendência: Se houver uma indicação } \\
\text { de como lidar }\end{array}$ & Não & Não & Não \\
\hline Controle na componente Horizontal & Sim & Sim & Sim \\
\hline Controle na componente Vertical & Sim & Sim & Sim \\
\hline $\begin{array}{c}\text { Desvio-padrão é a medida de } \\
\text { acurácia proposta }\end{array}$ & Não & Não & Não \\
\hline RMS é a medida de acurácia proposta & Sim & Sim & Sim \\
\hline Normalidade explícita & Sim & Não & Sim \\
\hline $\begin{array}{c}\text { Nível de confiança: Nível de } \\
\text { probabilidade considerado para o } \\
\text { resultado da avaliação }\end{array}$ & $95 \%$ & $90 \%$ & $100 \%$ \\
\hline Relatório de Controle de Qualidade & Não & Sim & Sim \\
\hline $\begin{array}{c}\text { Circular/linear: se as componentes } \\
\text { planimétricas (XY) são analisadas em } \\
\text { conjunto (circular) ou } \\
\text { independentemente (linear) }\end{array}$ & Circular & Circular & Circular \\
\hline Exemplo de aplicações & Não & Sim & Não \\
\hline
\end{tabular}

Fonte: Os autores (2021).

\section{CONCLUSÃO}

De acordo com esse estudo verifica-se que a componente posicional é fundamental e de grande importância no processo de avaliação da acurácia posicional de produtos cartográficos, sendo imprescindível a sua análise, principalmente, em trabalhos que requerem alta precisão.

Diante dos resultados obtidos pode-se perceber a diferença entre cada norma, e apesar de utilizarem a estatística, as mesmas se diferem bastante quando o assunto é determinar o tamanho amostral. Além disso, somente a norma de execução $\mathrm{n}^{\circ} 02$ de INCRA é a única que deixa de forma explícita como deve-se proceder a detecção de tendência, apesar de não dizer o que deve ser feito no caso de haver esses efeitos sistemáticos no produto.

Outra questão é a detecção de outliers, nenhuma das normas detalha se deve ser feita essa verificação. Já em relação ao padrão de distribuição amostral, nada é abordado nessas normas, apenas é dito que a amostra 
deve estar bem distribuída pela área de estudo.

Portanto, é importante destacar a relevância das pesquisas nas normas do controle de qualidade em produtos cartográficos, assim como, a aplicação da metodologia proposta por Santos et al., (2016) no qual permite a avaliação da acurácia posicional de um produto cartográfico desde a amostragem até a classificação final, utilizando-se para isso de alguns conceitos de estatística espacial.

Toda esta metodologia, guia deste trabalho, foi desenvolvida com o auxílio do software GeoPEC v3.5.2. Este programa auxilia no processo e é de fácil utilização, entretanto, cabe ressaltar que sempre será necessário um profundo conhecimento, senso crítico e discernimento do analista responsável pelo procedimento da avaliação.

Dessa forma, é notório que ambas as normas necessitam de uma melhoria em suas metodologias, para que todo esse processo seja feito de forma satisfatória e com rigor, sem que haja dúvidas de sua aplicação, visando tanto o produtor, bem como o usuário.

\section{Agradecimentos}

Os autores agradecem ao incentivo da Universidade Federal de Viçosa através do Departamento de Engenharia Civil, curso de Pós-Graduação em Engenharia Civil, na realização deste trabalho, além do apoio da Coordenação de Aperfeiçoamento de Pessoal de Nível Superior - Brasil (CAPES) - Código de Financiamento 001. Agradecimentos ao Instituto Nacional de reforma Agrária (INCRA) pela disponibilização dos dados utilizados.

\section{Contribuição dos Autores}

Todos os autores tiveram contribuições essenciais para elaboração deste artigo. Conceptualização, Curadoria dos dados, Análise formal, Investigação, Metodologia, Recursos, Software, Supervisão, Validação, Redação (revisão e edição) teve colaboração de todos os autores. A Redação (minuta inicial) teve participação principal do primeiro e segundo autor. A administração do projeto teve participação principal do quarto autor. O aerolevantamento e a geração da ortofoto ficou a cargo do terceiro autor.

\section{Conflitos de Interesse}

Os autores declaram que não há conflitos de interesse.

\section{Referências}

ALMEIDA, I. C. et al. Estudo sobre o uso de veículo aéreo não tripulado (VANT) para mapeamento aéreo com fins de elaboração de projetos viários. In: VI Simpósio Brasileiro de Ciências Geodésicas e Tecnologias da Geoinformação, 2016, Recife. Anais...Recife: UFPE, 2016. p. 1-10.

ARIZA, F.J; ATKINSON, A. D. Analysis of Some Positional Accuracy Assessment Methodologies. Journal of Surveying Engineering 134 (2). American Society of Civil Engineers: p. 45-54. 2008.

\section{AMERICAN SOCIETY FOR PHOTOGRAMMETRY AND REMOTE SENSING (ASPRS). ASPRS} Accuracy Standards for Digital Geospatial Data, pp. A1-A26, 2014. Disponível em: <http://www.asprs.org/a/society/divisions/pad/Accuracy/Draft_ASPRS_Accuracy_Standards_for_Digita 1_Geospatial_Data_PE\&RS.pdf $>$. Acesso em: 10 jan. 2021.

BRASIL. Decreto $\mathbf{N}^{\mathbf{0}} \mathbf{8 9 . 8 1 7}$ de 20 de junho de 1984. Normas Técnicas da Cartografia Nacional. Brasil, 1984.

BRASIL. Decreto $n^{\circ}$ 6.666, de 27 de novembro de 2008. Institui a Infraestrutura Nacional de Dados Espaciais - INDE, e dá outras providências. Diário Oficial da União. Retirado de <http://www.planalto.gov.br/ccivil_03/_Ato2007-2010/2008/Decreto/D6666.htm>. Acessado em: 07 de outubro de 2019. 
CARVALHO, J. A. B.; SILVA, D. C. Métodos para avaliação da acurácia posicional altimétrica no Brasil. Revista Brasileira de Cartografia (online), vol. 70, n. 2, pp. 725-744, 2018.

CÂNDIDO, A. K. A. A.; DA SILVA, N. M.; FILHO, A. C. P. Imagens de alta resolução espacial de Veículos Aéreos Não Tripulados (VANT) no planejamento do uso e ocupação do solo. Anuário do Instituto de Geociências, v. 38, n. 1, p. 147-156, 2015.

DIRETORIA DE SERVIÇO GEOGRÁFICO (DSG). Especificação Técnica Para Aquisição de Dados Geoespaciais Vetoriais (ET-ADGV). Brasil, 2011. Retirado de: <http://www.geoportal.eb.mil.br/images/PDF/ET_ADGV_Vs_2_1_3.pdf>. Acessado em: 01 de outubro de 2019.

DIRETORIA DE SERVIÇO GEOGRÁFICO (DSG). Especificação Técnica para Controle de Qualidade de Dados Geospaciais (ET-CQDG). Brasil, 2016. Retirado de: <http://www.geoportal.eb.mil.br/images/PDF/ET_CQDG_1a_edicao_2016.pdf〉. Acessado em: 01 de outubro de 2019.

GEOPEC. Software para avaliação da acurácia posicional em dados cartográficos. Disponível em: <http://www.geopec.com.br/p/software-geopec.html>. Acesso em 5 jan. 2021.

INSTITUTO NACIONAL DE COLONIZAÇÃO E REFORMA AGRÁRIA (INCRA). Manual Técnico de Posicionamento. Distrito Federal, 2013. Disponível em: <https://metrica.zendesk.com/hc/ptbr/articles/203840955-Manual-T\%C3\%A9cnico-de-Posicionamento-1\%C2\%AAEdi\%C3\%A7\%C3\%A3o-da-NTGIR-INCRA>. Acesso em: 5 jan. 2021.

INSTITUTO NACIONAL DE COLONIZAÇÃO E REFORMA AGRÁRIA (INCRA). Norma de Execução do INCRA $\mathbf{n}^{\circ}$ 02. Distrito Federal, 02 p., 2018. Disponível em: <https://mundogeo.com/wpcontent/uploads/2018/02/NE-INCRA-DF-N-02.pdf>. Acesso em: 10 jan. 2021.

INTERNATIONAL ORGANIZATION FOR STANDARDIZATION (ISO). Sampling procedures for inspection by attributes - Part 2: Sampling plans indexed by limiting quality (LQ) for isolated lot inspection, 1985. Disponível em: <https://www.iso.org/standard/64505.html>. Acesso em: 5 jan. 2021.

INTERNATIONAL ORGANIZATION FOR STANDARDIZATION (ISO). Sampling procedures for inspection by attributes - Part 1: Sampling schemes indexed by acceptance quality limit (AQL) for lot-by-lot inspection, $1999 . \quad$ Disponível em: <https://www.3ppars.com/WebsiteImages/download/4945362907.PDF>. Acesso em: 5 jan. 2021.

INTERNATIONAL ORGANIZATION FOR STANDARDIZATION (ISO). Geographic information - Data quality, 2013. Disponível em: <https://www.iso.org/standard/32575.html>. Acesso em: 5 jan. 2021.

HOLLER, W. A.; SERRATO, R. J.; GRAÇA, N. L. S. S.; CRUZ, W.; CRISTO, D. P. Avaliação de acurácia e precisão de modelos digitais de superfície a partir de diferentes aeronaves remotamente pilotadas. Revista Técnico Científica do CREA-PR, 13a ed., 20 p., 2018.

LISBOA, M. H.; SANTOS, A. P.; MEDEIROS, N. DAS G.; ABREU, M. V. S. Avaliação da Acurácia Posicional Tridimensional de Produtos Cartográficos Utilizando um Elipsoide de Incertezas. Revista Brasileira de Cartografia, v. 71, n. 4, p. 1040-1063, 13 dez. 2019.

NERO, M. A. Proposta para o controle de qualidade de bases cartográficas com ênfase na componente posicional. Tese de doutorado. Escola Politécnica da Universidade de São Paulo, Programa de PósGraduação em Engenharia de Transportes, São Paulo,186 p., 2005.

FONSECA NETO, F. D. et al. Avaliação da qualidade posicional de ortofotos geradas por SISVANT com diferentes alturas de voo para aplicações cadastrais. Revista Brasileira de Cartografia, v. 69, n. 7, 2017.

PEREIRA, T. A. J.; NERO, M. A. Análise de normas de controle de qualidade posicional em cartografia ao redor do mundo: exemplos atuais de alguns países. In: IV Simpósio Brasileiro de Ciências Geodésicas e Tecnologias da Geoinformação, 2012, Recife. Anais... Recife:UFPE, 2012, p. 01-09. 
PONTES, D. F. et al. Uso de Aeronave Pilotada a Distância para el análisis de Inundaciones: Un Estudio de Caso del Complejo Paso. REDER, v. 4, n. 2, p. 97-110, 2020.

RESENDE, D. B. et al. Uso de imagens tomadas por aeronaves remotamente pilotadas para detecção da cultura do milho infestada por Spodoptera frugiperda. Revista Brasileira de Geografia Física, v. 13, n. 01, p. 156-166, 2020.

SANTOS, A. P. Avaliação da Acurácia Posicional em Dados Espaciais com o Uso de Estatística Espacial. Dissertação (Mestrado em Engenharia Civil). Viçosa: Universidade Federal de Viçosa, 128 p., 2010.

SANTOS, A. P. RODRIGUES, D. D. SANTOS, N. T. GRIPP JÚNIOR, J. Avaliação da Acurácia Posicional em Dados Espaciais Utilizando Técnicas de Estatística Espacial: Proposta de Método e Exemplo Utilizando a Norma Brasileira. Boletim de Ciências Geodésicas, v.22, n.4, p.630-650, 2016.

SAITO, Y. K.; VIANA, L. J. F.; SILVA, S. R. DA; FONSECA, I. G. R. DA; SANTOS, A. DE P. DOS. Métodos Distância de Hausdorff e Influência do Vértice a partir da densificação de feições lineares. Revista Brasileira de Cartografia, v. 71, n. 2, p. 598-618, 27 jun. 2019.

ZANETTI, J.; BRAGA, F. L. S.; DOS SANTOS, A. DE P. Comparativo das normas de controle de qualidade posicional de produtos cartográficos do Brasil, da ASPRS e da OTAN. Revista Brasileira de Cartografia, v. 70, n. 1, p. 359-390, 2018.

\section{Biografia do autor principal}

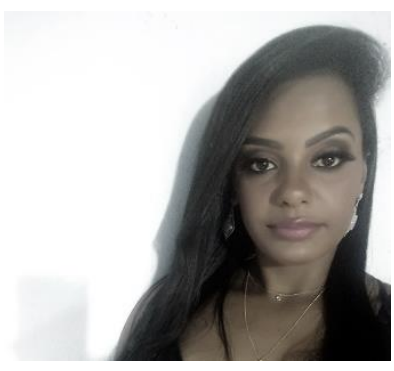

Lígia da Silva Barbosa nasceu em Visconde do Rio Branco-MG em 1996. Possui graduação em Engenharia de Agrimensura e Cartográfica pela Universidade Federal de Viçosa (2019) e atualmente é mestranda em Engenharia Civil, área de Concentração em Informações Espaciais (Cartografia) pela Universidade Federal de Viçosa. Pesquisa tendo foco principal o controle de qualidade em produtos cartográficos. 